\title{
Salvage endoscopic submucosal dissection with a small-caliber endoscope for recurrent esophageal cancer on distal side of esophageal stricture
}

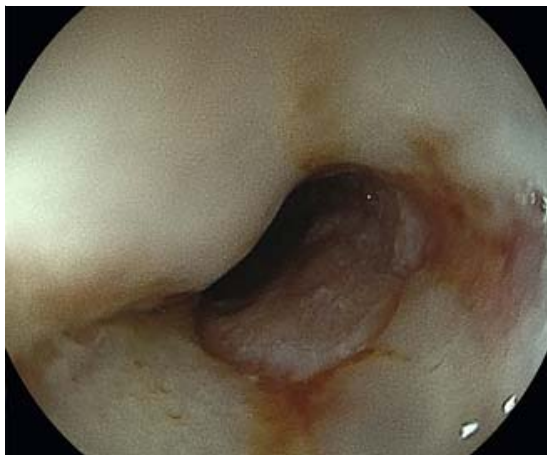

- Fig. 1 Endoscopy showed circumferential ulceration and stricture in the cervical esophagus that had developed due to chemoradiotherapy.

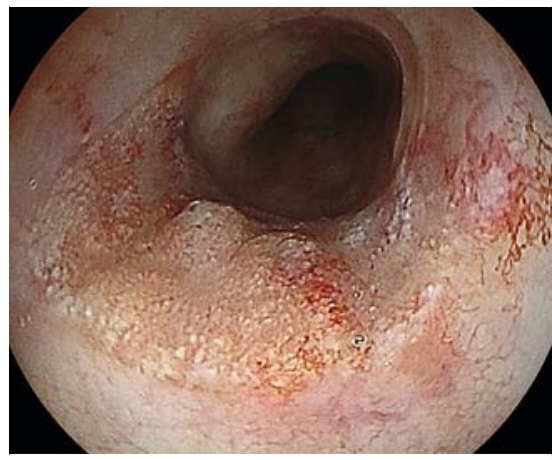

Fig. 2 Endoscopy showed a half-circumferential flat cancer on the distal side of the esophageal stricture.

Salvage endoscopic submucosal dissection (ESD) is effective for local recurrent esophageal cancer after chemoradiotherapy [1, 2]. However, severe late toxicity, mostly in the form of esophageal strictures and ulcerations, can occur in patients who undergo chemoradiotherapy in the definitive treatment of esophageal cancer [3]. Endoscopic treatment for esophageal neoplasia in patients with esophageal stricture is challenging [4].

A 76-year-old woman with recurrent esophageal cancer after chemoradiotherapy was referred to our department for endoscopic treatment. Endoscopy showed a cervical esophageal stricture

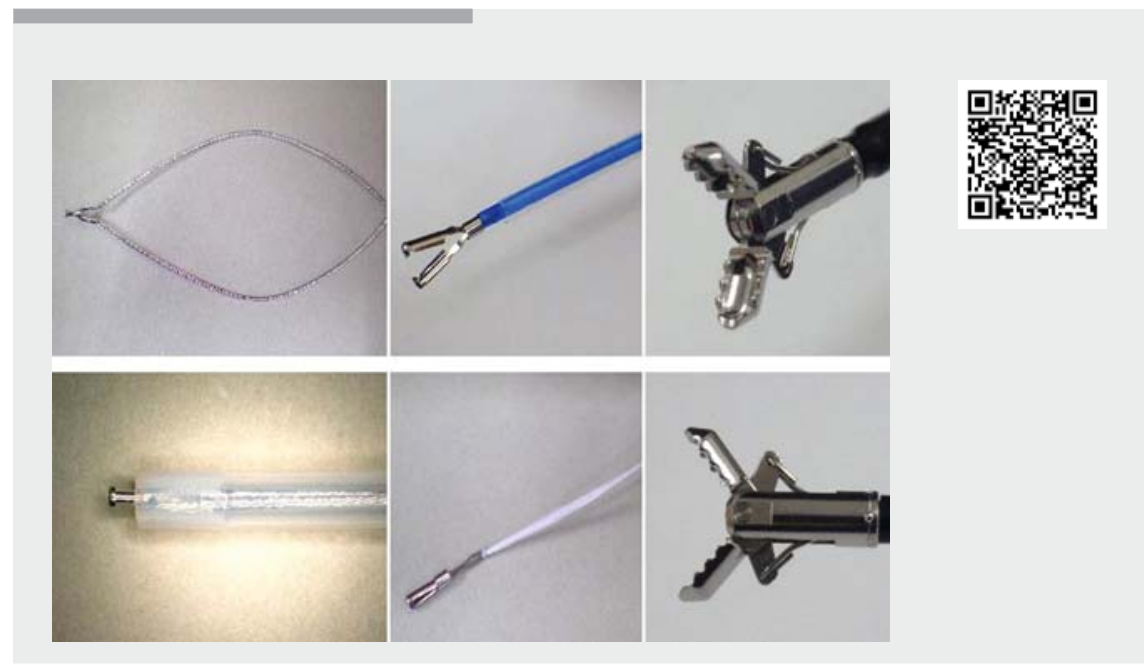

Video 1 Endoscopic submucosal dissection with a small-caliber endoscope for recurrent esophageal cancer on the distal side of the esophageal stricture.

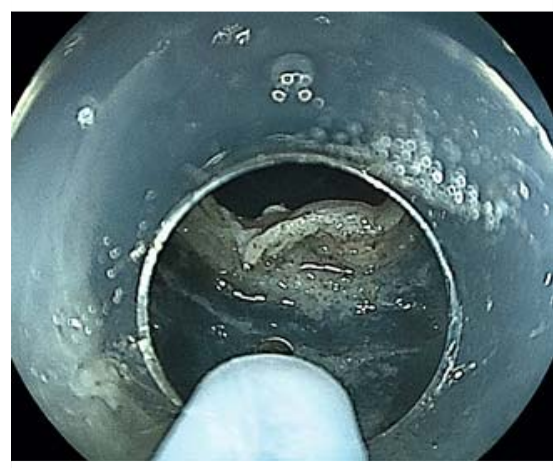

- Fig. 3 Markings, mucosal incision, and submucosal dissection were all performed using an endo-knife.

due to chemoradiotherapy ( $\triangleright$ Fig. $\mathbf{1}$ ) and a half-circumferential flat cancer on the distal side of the stricture ( $\downarrow$ Fig. 2; - Video 1). Although balloon dilation was performed, the therapeutic endoscope could not be passed through the stricture. We performed ESD with a small-caliber endoscope (EG-L580NW7; Fujifilm, Tokyo, Japan; diameter $5.8 \mathrm{~mm}$, working channel $2.4 \mathrm{~mm}$ ). Small-caliber ESD devices such as an endo-knife, clipline traction device, and hemostatic for-

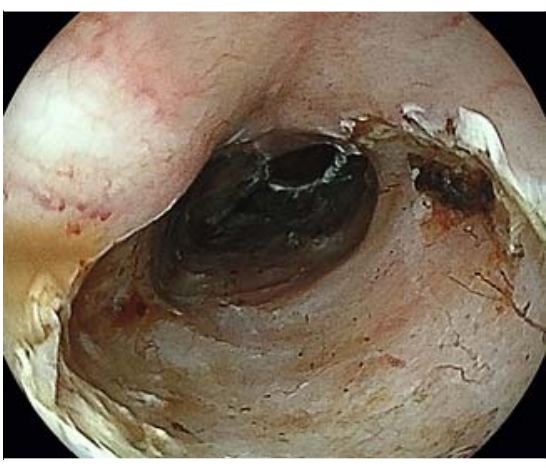

- Fig. 4 The lesion was completely resected en bloc in 55 minutes without any complications.

ceps were used (Souten, Ichigan, and Raicho, respectively; Kaneka Medics, Tokyo, Japan). A distal attachment was used to stabilize the endoscope. Markings, mucosal incision, and submucosal dissection were all performed using the endo-knife (> Fig.3). After circumferential mucosal incision, a traction device was applied to obtain a good field of view for dissection. A small-caliber hemostatic forceps was used to arrest bleeding. Finally, the lesion was comple- 

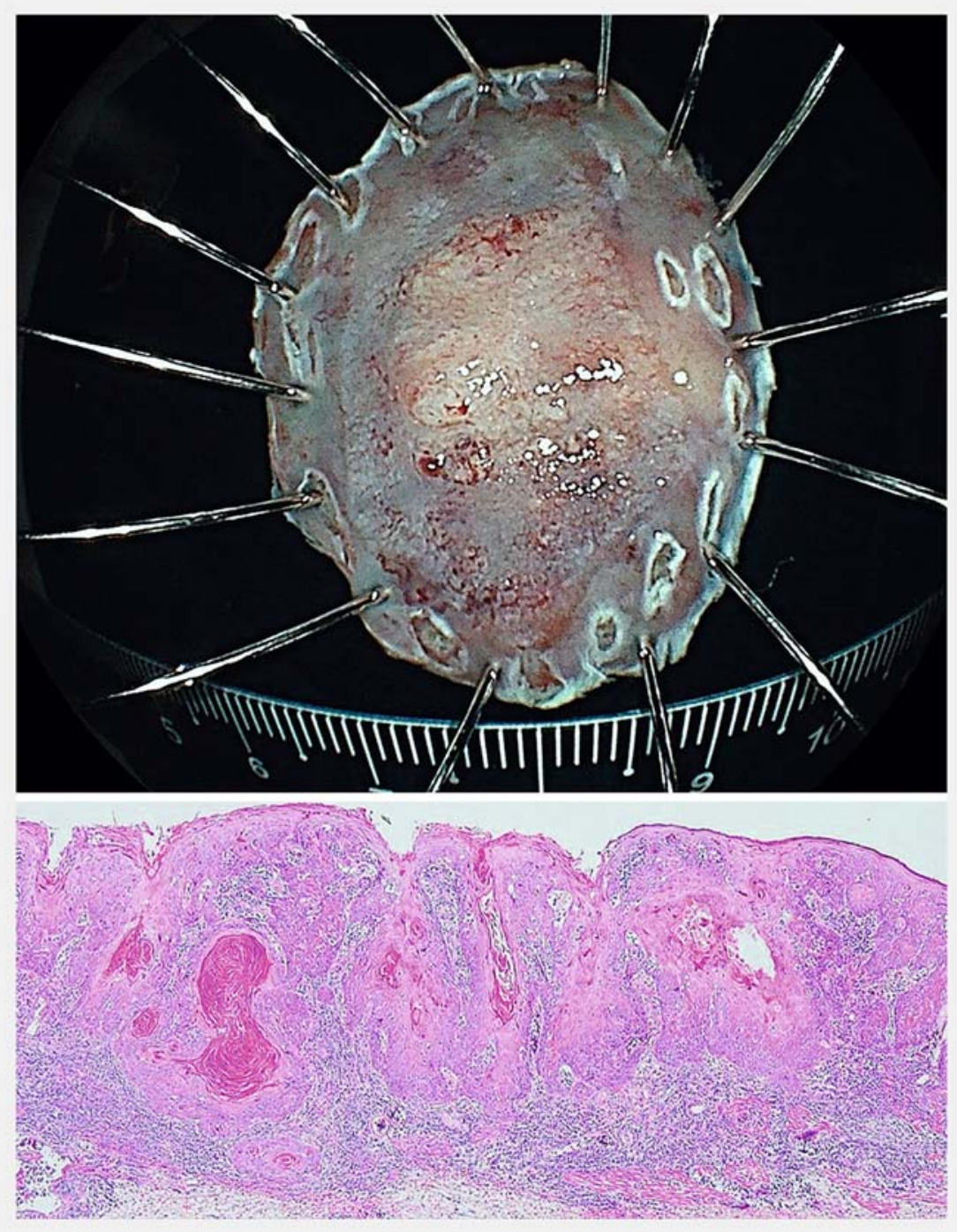

- Fig. 5 Histopathological examination revealed squamous cell carcinoma with slight submucosal invasion and negative horizontal and vertical margins. Upper: macroscopic view of the resected specimen. Lower: hematoxylin-eosin stain, $\times 100$ magnification.

tely resected en bloc ( $\$$ Fig. 4 ) in 55 minutes without any complications. Histopathology revealed squamous cell carcinoma with slight submucosal invasion and negative horizontal and vertical margins ( $\downarrow$ Fig.5). No other treatment for the recurrent esophageal cancer was performed because the patient refused additional surgical treatment.

In conclusion, salvage ESD with a smallcaliber endoscope can be a treatment option for recurrent superficial esophageal cancer after chemoradiotherapy

\section{Competing interests}

The authors

Kyosuke Tanaka', Yuhei Umeda ${ }^{1,2}$, Hiroki Asakawa ${ }^{2}$, Hiroshi Miura ${ }^{1}$, Misaki Nakamura ${ }^{2}$, Masaki Katsurahara ${ }^{1}$, Yasuhiko Hamada ${ }^{2}$

1 Department of Endoscopy, Mie University Hospital, Tsu, Japan

2 Department of Gastroenterology and Hepatology, Mie University Hospital, Tsu, Japan

\section{Corresponding author}

\section{Kyosuke Tanaka, MD, PhD}

Department of Endoscopy, Mie University Hospital, 2-174 Edobashi, Tsu, Mie 5148507, Japan

Fax: +81-59-231-5200

kyosuket@clin.medic.mie-u.ac.jp

\section{References}

[1] Hatogai K, Yano T, Kojima T et al. Local efficacy and survival outcome of salvage endoscopic therapy for local recurrent lesions after definitive chemoradiotherapy for esophageal cancer. Radiat Oncol 2016; 11: 31

[2] Saito Y, Takisawa H, Suzuki H et al. Endoscopic submucosal dissection of recurrent or residual superficial esophageal cancer after chemoradiotherapy. Gastrointest Endosc 2008; 67: 355-359

[3] Roeder F, Nicolay NH, Nguyen T et al. Intensity modulated radiotherapy (IMRT) with concurrent chemotherapy as definitive treatment of locally advanced esophageal cancer. Radiat Oncol 2014; 9: 191

[4] Nishiyama N, Kobara H, Yachida T et al. Strategy of small-caliber endoscopic submucosal dissection for esophageal neoplasia distal to severe stricture. Endoscopy 2020; 52: E94-E95

where a conventional endoscope cannot be used because of proximal esophageal stricture.

Endoscopy_UCTN_Code_TTT_1AO_2AG

The authors declare that they have no conflict of interest.
Endoscopy 2021; 53: E363-E364

DOI 10.1055/a-1296-7632

ISSN 0013-726X

published online 19.11.2020

(c) 2020. Thieme. All rights reserved. Georg Thieme Verlag KG, Rüdigerstraße 14, 70469 Stuttgart, Germany 